Journal of Research in Interprofessional

Practice and

Education

Vol. 4.2

October 2014

Journal of Research in Interprofessional Practice and Education (JRIPE)

Vol. 4.2

(c) 2014

Corresponding author: Paul Nicol. Email: ipnicol@iinet.net.aü.

\section{Attributes of Effective Interprofessional Placement Facilitation}

\author{
Paul Welby Nicol, PhD \& Dawn Forman, PhD
}

\begin{abstract}
Background: The quality of facilitation is an important influence on the efficacy of interprofessional education (IPE) delivery. The research objective was to increase understanding of the attributes of effective facilitation of students during external IPE placements in primary care situations.

Methods and Findings: A thematic analysis of the experiences of academics, students, and placement-site staff at three placement sites was employed to explore participants' perceptions of the attributes of effective IPE facilitators. These attributes included experience in an interprofessional context, together with an understanding of the specific clinical and assessment requirements of different disciplines. Facilitators also needed empathy with respect to the requirements of the external IPE placement sites and the ability to liaise between student and site needs.

Conclusions: Models of IPE placement facilitation were most effective when, while following general principles, facilitators tailored them specifically for the individual situations of the placement sites and the learning requirements of particular groups of students. The most rewarding IPE learning experiences occurred when IPE facilitators provided sufficient clinical opportunities for students to work collaboratively with individual clients, provided the students perceived that their participation was relevant to their own discipline.

Keywords: Interprofessional education; Interprofessional practice; Facilitation; Placements
\end{abstract}

\section{Introduction}

The quality of facilitation has been shown to be an important factor influencing the effectiveness of interprofessional education (IPE) delivery [1] in instilling lasting interprofessional practice (IPP) attitudes in students. As well, Ponzer et al. [2] have shown that students' satisfaction with facilitation may be positively correlated with their attitudes toward an IPE placement. Conversely, inadequate facilitation can result in negativity toward collaborative practice [3], especially when professional boundaries and identities can be challenged, as in IPE [4]. With this in mind, Newell-Jones and Lord [5] identified a need to improve the quality of IPE facilitation, through more training.

Chipchase, Allen, Eley, McAllister, and Strong [6], based on a literature review, have suggested that, in addition to supervision qualities of inspiration, reassurance, and support, IPE facilitators need an understanding of team formation and effectiveness, group dynamics, and conflict resolution. In addition, Reeves et al. [1] included practical skills; experience, especially with interprofessional collaboration; knowledge and understanding of the professions and their current issues; confidence to meet the differing demands of an interprofessional group; and a good understanding of group learning theories. A teamwork focus has been identified as another important attribute of IPE facilitation [7]. 
2

IPE Placement Facilitation

Nicol \& Forman

Journal of Research in Interprofessional Practice and Education

Vol. 4.2

October 2014
This article explores and extends these attributes in the specific context of IPE placements, based on experience at off-campus IPE placement sites. In 2011, a Western Australian university, supported by grant funding from the governmentbacked Health Workforce Australia, increased the number of opportunities for students to experience IPE placements. These sites included residential aged care, primary and pre-primary schools, mental health, a hospital-based student training ward, and on-campus clinics [8].

Traditionally, discipline-specific supervision on clinical placements has been provided by clinicians in situ. For IPE placements, by contrast, the experience at these sites has been that it is not feasible to provide resources for clinicians from each and every discipline to be available continuously on site. Instead, the full-time presence of IPE facilitators was supplemented with regular on-site visits by discipline-specific supervisors.

The university has developed a program to prepare its IPE facilitators [9] based on its experience, starting with a 2009 pilot placement [10]. The program includes IPE competencies. However, this article is concerned with what the research participants themselves identified as attributes of good IPE facilitation, rather than the prescribed IPE competencies. For this research, the definition of IPE as occurring "when two or more professions learn with, from and about each other to improve collaboration and quality of care" [11] was adopted. IPP occurs when the results of this learning are put into practice in the health workplace and in the community [12].

\section{Method}

The objective was to increase understanding of the attributes of effective facilitation of students during external IPE placements in primary care situations. To achieve this, experiences of university and site staff, as well as students, were explored.

The research to identify these attributes was an extension of a qualitative research project that was designed to contribute toward evaluation of the first four years of an IPE program at the university ("IPE evaluation") (unpublished). Placements at the university were evaluated using a modified University of West of England instrument, the UWE Interprofessional Questionnaire [13], in which questionnaires are used to explore students' attitudes toward IPE, together with their communication and teamwork skills and interprofessional relationships. In addition, students' development of interprofessional capabilities, particularly for final year students, was measured using the faculty's Interprofessional Capabilities Assessment Tool (ICAT) [14]. The qualitative IPE evaluation was designed to complement these quantitative evaluations by exploring in depth the perspectives of participants in the IPE experience. The importance of IPE placement facilitation was one of the themes that emerged from it.

For the IPE evaluation, a modified grounded theory method [15] was adopted. A selective sample of 48 participants was recruited by invitation through email or word of mouth, based on their experience in the IPE program, to provide informed insights from a wide variety of perspectives. Student participants had little or no previous IPE exposure. Semi-structured interviews and focus groups were audio- 


\section{JRIPE}

3

IPE Placement Facilitation

Nicol \& Forman

Journal of Research in Interprofessional Practice and Education

Vol. 4.2

October 2014
Journal of Research in Interprofessional Practice and Education

recorded to provide validation for the notes used for coding. The coding vehicle used for the iterative process of constant comparison was the NVivo qualitative analysis software package.

\section{Figure 1 \\ Interview and focus group guides}

Interviews

The guide for the semi-structured interviews for the IPE evaluation was tailored, within an overall framework, to individual participants based on their position and experience. Also, in accordance with grounded theory principles, it evolved as the iterative process of constant comparison continued. This is a brief outline of the generic interview guide.

1. Understanding of the terms IPE and IPL

2. Role in IPE

3. Reasons for interest/involvement in IPE

4. Factors affecting the implementation of IPE placements

5. Barriers that have been overcome and how

6. Outcomes of the IPE initiative

7. The effect of the IPE on staff at the university and at placement venues

8. Student feedback on IPE

9. The future of IPE and lessons from participant's experience so far

10. Anything else participant wanted to discuss

\section{Focus groups}

Generic focus group questions were framed from the following guide:

1. What difference IPE placements make, compared with disciplinespecific placements

2. In what ways being involved with IPE placement affects work/learning

3. Benefits and drawbacks of IPE

4. How IPE placements can be improved

One strong emergent concept from the IPE evaluation constant comparison was the importance of IPE placement facilitation. As the data on this theme were particularly rich, it was subjected to a further in-depth exploration of participants' perspectives on the attributes of an effective IPE facilitator. For this further exploration a thematic approach was used, employing the NVivo software to group concepts for the emergence of the themes. The analysis was conducted by a single researcher, and the emergent themes were checked for fit, relevance, and workability [16] by a second, with any adjustments made by consensus. Included in this analysis were 20 of the IPE evaluation interviews - all those where IPE placements had been discussed- 


\section{JRIPE}

4

IPE Placement Facilitation

Nicol \& Forman

and the focus groups, which involved 13 participants. An analysis of the types of participants in both studies appears in Table 1.

Table 1

\section{Participants in the study}

\begin{tabular}{|l|c|c|}
\hline Interviewees & $\begin{array}{c}\text { IPE facilitation } \\
\text { analysis }\end{array}$ & $\begin{array}{c}\text { IPE evaluation } \\
\text { participants }\end{array}$ \\
\hline University staff & 12 & 18 \\
\hline University student & 0 & 1 \\
\hline External academic & 0 & 1 \\
\hline External health professional & 0 & 1 \\
\hline IPE site principals & 4 & 4 \\
\hline IPE site staff & 4 & 4 \\
\hline Parents of school children & 0 & 4 \\
\hline Focus groups (2) & & \\
\hline Students & 7 & 7 \\
\hline IPE site staff & 6 & 6 \\
\hline
\end{tabular}

The IPE placement sites included in both the IPE evaluation and the IPE facilitation attributes studies were two aged-care residential facilities and one school catering to primary and pre-primary children. They were selected because they were considered to be the sites that had the longest experience of IPE placements. At one of the aged-care sites, IPE placements were conducted in collaboration with medical students from another university. Student placements varied in length from a few days to 11 weeks, depending on the requirements of their discipline. Students experienced interprofessional clinical work and collaborative projects and activities on site.

The IPE facilitators were usually from a different discipline than most of their students. Discipline-specific supervisors complemented the IPE facilitation with regular visits to the site on a part-time basis. At one site, the IPE facilitator also provided the supervision for an individual discipline.

The study was approved by the Curtin University Human Research Ethics Committee and all participants gave informed consent that complied with the university ethics protocols.

\section{Findings}

Journal of Research in Interprofessional Practice and Education

Vol. 4.2

October 2014

\section{Facilitator's role}

There was a consensus that a high quality of IPE facilitation was vital for students to obtain a meaningful and worthwhile experience on a placement.

For this, IPE facilitators needed to ensure that each placement group consisted of a combination of disciplines and student experience that could work well together, and that the experiences were appropriate for that particular group of students. 
5

IPE Placement Facilitation

Nicol \& Forman
Journal of Research in Interprofessional Practice and Education

Vol. 4.2

October 2014
Students stated that they considered the best learning experience was when there was a range of several-four or five, rather than, say, two-disciplines collaborating on appropriate tasks.

However, participants considered that the principal role of an IPE facilitator was, as explained by one facilitator:

teaching students the "soft" skills-behaviour management, how to talk to a teacher or a parent in a parent-friendly way, how to write notes professionally, how to problem solve and resolve conflicts, how to structure a group session, how to work together-all non-disciplinary skills. This complements the discipline supervision well, because they are things the discipline-specific supervisors want their students to know, but don't have time to teach.

These soft skills, as compared to "hard" clinical skills, were developed by students working independently in self-directed groups with clients and on projects, with the IPE facilitators' role largely consisting of modelling collaborative practice, mentoring, and debriefing. The IPE facilitators assessed broad generic skills like communication and how students related to people, and they ensured students were following curricula to meet course accreditation requirements.

In addition, for students to achieve a good learning outcome, IPE facilitators needed to ensure the experience maintained student interest. An important part of this was ensuring that students received sufficient clinical opportunities with individual clients in an interprofessional context. Most participants considered clinical practice to be the most important IPE learning experience.

\section{Discipline relevance}

The facilitator needed to make sure that students worked together to decide what was appropriate for their client and then delivered the parts relevant to their profession. Students had to be able to see how what they were doing was relevant to their own discipline. Several participants stated that facilitators should avoid asking students to perform tasks that did not fall within the scope of their discipline or were not relevant to it.

Reflecting this, both the facilitator and academic participants stated that it was important for IPE placement facilitators to have some understanding of different disciplines, so that they could assist students to meet some of the discipline-specific requirements of the placement as well as the IPE aspects. This included, according to one participant:

making sure students have an opportunity to pass the prac, with regard to their different discipline-specific competencies and skills ... to tailor their time together accordingly ... as well as ... the chance to work together and get the collaborative experience.

For this, the facilitators needed at least an understanding of individual discipline course outlines. 
6

IPE Placement Facilitation

Nicol \& Forman

Journal of Research in Interprofessional Practice and Education

Vol. 4.2

October 2014

\section{Multidiscipline understanding}

Appreciation of the perspectives of different disciplines was essential not only to facilitate collaboration but also to address conflict resolution in the student teams. Divergent approaches of different disciplines stemmed from what was termed their different ways of thinking. One participant illustrated this with an opinion that a physiotherapist's perspective would be to concentrate on a client's pathology, whereas an occupational therapist would view what functions the client possessed that could be improved. Students who had been, in the words of one participant, "bombarded with the perspectives of their own disciplines" through their university life found their viewpoints were challenged by other students on IPE placements.

Several participants expressed the view that to reconcile such conflicting perspectives it was an advantage for IPE facilitators to have had experience working in an interprofessional setting. This experience would increase the likelihood of the facilitator exhibiting understanding of other professions' roles when co-ordinating students of different disciplines and personalities. One facilitator commented:

For people without the background, it would be an extremely difficult job. You can't overlook how much awareness you need to have of other disciplines and their scope of practice in order to be an IPE facilitator.

\section{Discipline-specific support}

The IPE facilitation was supplemented by visits to the IPE placement sites by discipline-specific supervisors. The frequency and duration of these visits varied according to the requirements of each discipline. Several site staff and students commented that it could increase IPE opportunities if the discipline-specific supervisor had an understanding of IPE. An academic said:

[if] discipline-specific supervisors know about IPE, they can talk about it with their students, for example, how what they have just taught the student would work well in collaboration with another professional.

Another suggestion was for students to be able to participate in supervisors' feedback to other students from different disciplines so they could hear the thought processes of that discipline.

Several student participants commented that they needed sufficient disciplinespecific support and supervision on IPE placements to teach and support them in developing their clinical skills in an interprofessional environment. However, in some cases they felt the supervisor was more concerned with assessing them. This could be disconcerting - "a bit nerve-wracking"-particularly if the supervisor had not been present to see what was happening from day to day at the IPE site.

Some students also commented that it was helpful if the visits of discipline-specific supervisors were synchronized, because when they were spending time with the discipline-specific supervisor they were withdrawn from IPE activities. The effect of scheduling discipline-specific visits at the same time would be to reduce disruption to collaborative activities. Placement site staff participants echoed this view. 
IPE Placement Facilitation

Nicol \& Forman

Journal of Research in Interprofessional Practice and Education

Vol. 4.2

October 2014
Journal of Research in Interprofessional Practice and Education

In some cases, discipline-specific supervision assessment of the IPE placements was necessary as part of course accreditation. Because there was usually no one on site to provide this for all disciplines involved, extra university resources and planning were required to provide the appropriate supervision. One participant suggested that some discipline-specific supervision could be conducted through videoconferencing or other communications technology, to avoid some of the need for discipline-specific supervisors to travel to placement sites.

\section{Preparation and availability}

For the university, there were also timetabling issues, which were exacerbated in some cases where discipline-specific placement opportunities had to be planned well in advance in collaboration with other universities.

Planning and preparation were also an important part of the facilitator's role. Some of the students said they needed induction material well in advance to prepare for an IPE placement, and several site staff said that it was important for students to know what to expect. Site staff, too, emphasized the need for timely planning and preparation, so that appropriate programs could be prepared for students.

Most participants agreed that an IPE facilitator should be continuously available on site for students, and that without this availability students would be likely to learn less. One participant commented that:

students can't learn about IPE without a facilitator. It is too high level and too complex and too much of a different thinking pattern to not have a facilitator. They are not ready early in the year to be independent. If they are left, they do nothing or they go home early. They are not going to be collaborating well and asking each other questions and taking care in their case loads if they don't have a supervisor.

In this view, students who were still preoccupied with learning their own discipline needed readily available guidance to develop the capacity to think about and ask questions about someone else's profession, or to work collaboratively. Student feedback to one IPE facilitator indicated that students often felt they did not have the confidence to think in an IPE way until they had been on a placement for some time.

However, it was recognized that IPE facilitators needed to ensure students were given independence and sensibility. A student stated that "it is better for students to work out solutions together rather than be given the answer from a facilitator." And a facilitator stated that "you don't expect fourth-year students to need someone hanging around them prompting them-that would defeat the purpose.... Once set, they pretty much run with things. But some do need a lot of checking."

\section{Site-specific adaptation}

The model of IPE facilitation varied depending on the circumstances at each site. At the school, a full-time facilitator appointed by the university also had a liaison role, identifying activities and clinical opportunities in line with the school's program, as well as supporting the students. This model was seen as providing continuity both 
8

IPE Placement Facilitation

Nicol \& Forman

Journal of Research in Interprofessional Practice and Education

Vol. 4.2

October 2014 for the IPE and for the school programs. At the time of the research, at one aged-care residential facility, site staff were providing the facilitation, working as a team, each part-time together with other duties, but ensuring continual availability for students. This model was seen as appropriate to provide flexibility when student numbers were fluctuating. The third site, where a mix of university and site staff had been used, reported that the effectiveness of the use of a single IPE facilitator depended on the mix of disciplines and the interprofessional experience of the facilitator.

Common to each of the three sites was the need for IPE facilitators to understand the culture and procedures of the facility. One site participant said that "someone who knows clients on site and residents and is interested in IPE is the best [facilitator] option for us." This was also important for continuity of care, which could be affected by turnover of students. This was the sole concern of parents of children at the school, who were all otherwise positive about the program.

The placement site participants stated that although their objective was to provide a positive learning experience, this had to be accommodated with the procedures of the site and its requirements for client care and safety. Consequently, the IPE facilitator needed to liaise closely and sensitively with the site. One facilitator stated that the role included "regular liaison, making sure what we do is on track with their [the site's] vision."

Student programs were adapted to suit the site's needs. However, activities, including clinical work in the students' disciplines, also needed to reflect the requirements of the students' courses, and part of the IPE facilitation role was managing that collaboration.

Also, facilitators needed enthusiasm and commitment to enlist the support of site staff, especially in the early days of the establishment of the site program. Part of obtaining support was through professional development sessions for site staff. Once a rapport had been established, the positive and welcoming attitude of site staff became an important factor in the student experience. As well, several participants were appreciative of the mutual learning that was occurring between site staff and students and academics with the encouragement of the IPE facilitator.

As an example of the rapport that developed, one participant spoke of the understanding that site staff exhibited in assisting when some students had struggled with communication difficulties with clients or in explaining their rationale. Another (site staff) participant discussed how she appreciated being able to liaise with the IPE facilitator when she felt a client needed observation and assessment.

Several participants commented that this liaison was more effective when the placement site had a previous record of working with the university, such as with discipline-specific placements, and/or an IPP culture.

Participants agreed that for students, opportunity for reflection was an important part of their learning.

\section{Discussion}

This research has identified several attributes that are required for effective facilitation of IPE student placements at aged-care and school sites based on the experiences of academics, staff, and students involved with the facilities. Because of the differences in site cultures and procedures and the diversity of health disciplines, for maximum 
9

IPE Placement Facilitation

Nicol \& Forman

Journal of Research in Interprofessional Practice and Education

Vol. 4.2

October 2014 effectiveness facilitators need experience, understanding, and empathy to be able to tailor models of facilitation to the individual needs of students from different disciplines within the requirements of the placement-site working environment. Students appear to gain most value when facilitators are able to ensure that the learning experience enables them to work meaningfully toward developing their competency in their own discipline by gaining experience in a collaborative interprofessional setting.

The findings reinforce the contention of Ponzer et al. [2] and Davidson, Smith, Dodd, Smith, and O'Loughlan [17] that a high standard of IPE facilitation on external placements is of paramount importance in achieving satisfactory outcomes. The research has identified from practical experience several attributes that the IPE facilitator requires in addition to the support requirements of clinical supervision roles identified by Chipchase, Allen, Eley, McAllister, and Strong [6]. These, as discussed by Reeves, Goldman, and Oandasan [1] and Freeth, Hammick, Scott, Koppel, and Barr [18], include the understanding that comes from prior interprofessional experience, particular practical skills, and the flexibility to reconcile divergent demands from different disciplines and organizational situations.

A strong emergent theme was the participants' conviction that an on-site supervisor needs more than an understanding of IPE issues [19,20]. It was evident that for a collaborative venture such as an IPE placement to succeed, the need for compromise between the requirements of different disciplines was required [21,22]. For facilitators, the capability and practical experience to understand and resolve the conflicting perspectives of the disparate disciplines and the placement sites themselves was imperative. While the goodwill required to achieve this was evident at all three sites in this research, it was a challenge for facilitators.

The key to meeting this challenge was not only understanding of the requirements of the different disciplines but also responsiveness to student needs. Students in this study came to an IPE placement with little previous IPE exposure beyond a short orientation program. They were concerned that the experience would be meaningful in terms of their own discipline. If they could see this relevance to themselves, they tended to find the whole IPE experience rewarding. IPE facilitators needed the empathy, knowledge, and experience to ensure student satisfaction in this. This was the reason that it was helpful if the facilitators had worked in settings where they had had exposure to and worked with other professions besides their own.

The facilitator also needed to be responsive to the needs of individual students at different levels of competency development [23]. Without appropriate facilitation, not only could some students struggle and lose interest, but the IPE experience for other students could also be affected as the collaborative effort faltered, resulting in the kind of negative attitude to IPE identified by Freeth and Reeves [3]. In the smallgroup environment of placements, an effective facilitator should be able to quite readily ascertain and address the individual needs of students. An attribute contributing to this was the appreciation of teamwork and group dynamics, identified by Reeves, Goldman, and Oandasan [1] and Thistlethwaite [7], particularly to ensure student groups were appropriately constituted to be able to work well together and to mentor them through suitable cases or projects. 
10

IPE Placement Facilitation

Nicol \& Forman
Journal of Research in Interprofessional Practice and Education

Vol. 4.2

October 2014
The empathetic attributes of IPE facilitation extended to responsiveness to the requirements and organizational culture of the external IPE placement site, liaising to maintain a productive learning environment where student requirements could differ according to the combination of disciplines in the student groups. The flexibility needed for this was evident in the way the facilitators at each of the three sites adapted their approach according to both the site situation and the current combination of students from different disciplines. It was apparent that, within broad guidelines, each site required individual methods tailored to its situation, and that these could change over time and with different student groups.

This individualized approach to IPE placement facilitation was a key element in the quality of the student experience at each site. However, together with the need for supervision, assessment, and support of different disciplines at each IPE placement site, it is one of the factors that makes IPE placements resource intensive. Nevertheless, despite the resources challenges of this tailored approach, there is a strong belief that interprofessional practice will be an important part of efficient healthcare delivery in the future. The resources directed to IPE are an investment in addressing that need World Health Organization [24], and addressing the need for improvement in IPE facilitation identified by Newell-Jones and Lord [5] will help make better use of those resources. For IPE placements, this can be achieved by facilitators with the attributes described by the participants in the research reported in this article.

\section{Limitations}

The research was conducted in the context of IPE culture and practice within one university and three external placement sites. It is qualitative in methodology, so quite different perspectives may be obtained in other contexts. Nevertheless, the outcomes may be evaluated for their transferability to other situations where the experience of the participants may be of value.

\section{Conclusion}

This study has provided insights to advance knowledge of providing a quality IPE experience for students in a practical setting. Based on the perspectives of people engaged at different levels of the experience-academics, site staff, students, and parents of children-the research has provided empirical evidence of the qualities needed for effective placement facilitation that may be adopted and adapted for other contexts. Similar future studies in other contexts would further this knowledge. In addition, in future years, longitudinal studies to assess the impact of IPE on IPP in recently graduated health professionals would help in establishing the value of IPE.

\section{References}

1. Reeves, S., Goldman, J., \& Oandasan, I. (2007). Key factors in planning and implementing interprofessional education in health care settings. Journal of Allied Health, 36(4), 231-235.

2. Ponzer, S., Hylin, U., Kusoffsky, A., Lauffs, M., Lonka, K., Mattiasson, A.C., \& Nordström, G. (2004). Interprofessional training in the context of clinical practice: Goals and students' perceptions on clinical education wards. Medical Education, 38(7), 727-736.

3. Freeth, D., \& Reeves, S. (2004). Learning to work together: Using the presage, process, product (3P) model to highlight decisions and possibilities. Journal of Interprofessional Care, 18(1), 43-56. 
11

IPE Placement Facilitation

Nicol \& Forman

Journal of Research in Interprofessional Practice and Education

Vol. 4.2

October 2014
4. McCray, J. (2010). Interprofessional Collaboration for Change. In Scragg, T. (Ed.), Managing change in health and social care services. (pp. 149-160). Brighton: Pavilion Press.

5. Newell-Jones, K., \& Lord, M. (2008). A learning and teaching framework for interprofessional learning. In Howkins, E., \& Bray, J.A. (Eds.), Preparing for interprofessional teaching: Theory and practice. (pp. 13-26). Abingdon: Radcliffe Publishing.

6. Chipchase, L., Allen, S., Eley, D., McAllister, L., \& Strong, J. (2012). Interprofessional supervision in an intercultural context: A qualitative study. Journal of Interprofessional Care, 26(6), 465-471.

7. Thistlethwaite, J.E. (2012). Values-based interprofessional collaborative practice: Working together in health care (values-based practice) Cambridge, UK: Cambridge University Press.

8. Curtin Health Innovation Research Institute. (2012). Interprofessional student placements. URL: hittp://healthsciences.curtin.edu.au/local/docs Lebo.pdfín [August 23, 2012].

9. Curtin University. (2012). IPE practice facilitation preparation. URL: ihtttp://healthsciences.curting



10. Brewer, M., Franklin, D., \& Lawrence, J. (2011). Brightwater Care Group interprofessional place-

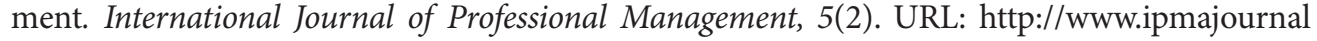
'com/articles/Vol5_Iss2_Article3.php' [November 26, 2011].

11. Centre for the Advancement of Interprofessional Education. (2002). Interprofessional education-A definition. URL:ihttp://www.caipe.org.uk/resources/defining-ipe/i[September 30, 2012].

12. Australasian Interprofessional Practice and Education Network. (n.d.). What is IPE/IPL/IPP? URL: htttp://wwwwaippen.net/what-is-ipe-ipl-ippi [September 30, 2012].

13. Pollard, K.C., Miers, M.E., \& Gilchrist, M. (2004). Collaborative learning for collaborative working? Initial findings from a longitudinal study of health and social care students. Health and Social Care in the Community, 12(4), 346-358.

14. Brewer, M., Gribble, N., Robinson, P., Lloyd, A., \& White, S. (2009). Assessment of interprofessional competencies for health professional students in fieldwork education placements. In Milton, J., Hall, C., Lang, J., Allan, G., \& Nomikoudis, M. (Eds.). ATN Assessment Conference 2009: Assessment in different dimensions. Conference proceedings. Melbourne: Learning and Teaching Unit, RMIT University.

15. Whiteley, A. (2002). Grounded research: A modifed grounded theory for the business setting. In Curtin University Graduate School of Business Working Paper Series 00.02. Perth, Australia: Graduate School of Business, Curtin University.

16. Glaser, B., (1998). Doing grounded theory: Issues and discussions. Mill Valley, CA: Sociology Press.

17. Davidson, M., Smith, R.A., Dodd, K.J., Smith, J.S., \& O’Loughlan, M.J. (2008). Interprofessional prequalification clinical education: A systematic review. Australian Health Review, 32(1), 111-120.

18. Freeth, D.S., Hammick, M., Scott, R., Koppel, I., \& Barr, H. (2005). Effective interprofessional education: Development, delivery and evaluation. London, UK: Blackwell Science.

19. Holland, K. (2002). Inter-professional education and practice: The role of the teacher/facilitator. Nurse Education in Practice, 2(4), 221-222.

20. Williamson, G.R., Callaghan, L., Whittlesea, E., Mutton, L., \& Heath, V. (2011). Placement development teams and interprofessional education with healthcare students. Journal of Clinical Nursing, 20(15-16), 2305-2314.

21. Haddad, A.R., Coover, K.L., \& Faulkner, M.A. (2011). Development and incorporation of an interprofessional experience into a geriatric pharmacy elective: The first-year experience. Currents in Pharmacy Teaching and Learning, 3(2), 116-122.

22. Steinert, Y. (2005). Learning together to teach together: Interprofessional education and faculty development. Journal of Interprofessional Care, 19(S1), 60-75.

23. D’Eon, M. (2005). A blueprint for interprofessional learning. Journal of Interprofessional Care, 19(S1), 49-59.

24. World Health Organization. (2010). World Health Organization framework for action on interprofes-

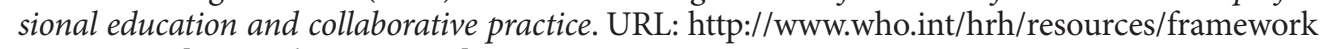
action_en_L_'_[September 18, 2012]. 